\title{
Multiscale Handwriting Characterization for Writers' Classification
}

\author{
Véronique Eglin, Stéphane Bres, and Carlos Rivero \\ LIRIS - INSA de Lyon, 20 avenue Albert Einstein, \\ 69621 Villeurbanne Cedex, France \\ \{veronique.eglin, carlos.rivero, stephane.bres\}@liris.cnrs.fr
}

\begin{abstract}
In this paper, we propose a method for handwritten text characterization based on a multiscale and multiresolution drawing analysis. The approach lies on the definition of four complementary handwritten text visual dimensions: the macro and micro orientation (obtained with a frequencies multiscale image analysis), the text linearity (defined by the merge of connected components), the curvature (measured as a multiresolution high profile deformation) and the complexity (expressed as multiscale drawing distribution entropy). Each feature is studied in an evolution graph that can be expressed as a unique handwritten curve signature. It leads to a description in separable writers families having individual visual characteristics. The results are very promising.
\end{abstract}

\section{Introduction}

Handwritten documents of $18^{\text {th }}$ and $19^{\text {th }}$ century authors like Montesquieu or Flaubert constitute rare collections that are preserved in libraries or specialized institutes and are recently associated to innovating digitalization projects. Among those manuscript collections from $18^{\text {th }}$ and $19^{\text {th }}$ century, we have been interested by an impressive, rare and complete collection of French manuscripts written by Montesquieu, a wellknown author of $18^{\text {th }}$ century. Montesquieu handwritten collection has been marked by intensive uses and manipulations that is expressed by a poor documents visual quality, with handwritten texts that are often erased with multi-writer annotations and corrections, see figure 1 .

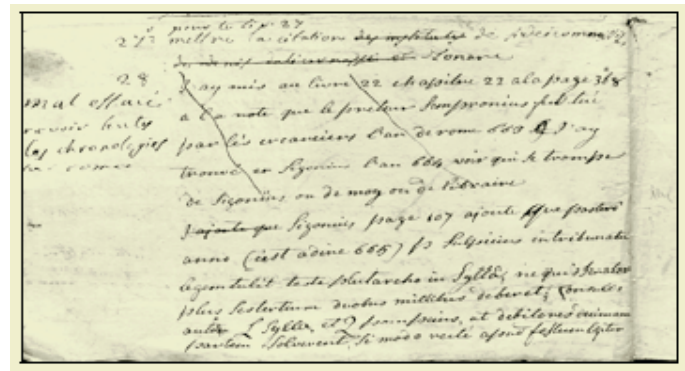

Fig. 1. Example of Montesquieu's autograph extracted from "De l'Esprit des Lois" (1789). 
Montesquieu manuscripts are characterized by a great diversity of writers: they were supported by more than twenty secretaries having all visual segregating characteristics of writings. Our purpose here is to prove that it is possible to identify different writers belonging to well identified writer's families by considering macroscopic visual characteristics with an analysis of writings forms based on the study of multiscale visual characteristics: the curve orientation, the drawing linearity, the multiresolution curvature and the global complexity as a measure of drawings density. In this study, we are trying to evaluate the writers' variability in a same page and in a same book. To do that, we propose a complete handwriting classification methodology into visual similar clusters. In the field of handwritten text recognition, we often consider the recognition task as a pure omni-writer problem, considering that letters and words can be processed independently and sequentially. We have chosen to consider handwritten text by considering visual emergent properties in the way of human visual expertise like Nosary in [5]. The originality of our approach is to consider a multiscale approach from a macroscopic (linked to the page dimension) to a microscopic (linked to the grapheme or the letter dimension) analysis. The macroscopic analysis leads on the consideration of texture properties of lines drawings, like it has been introduced by Kuckuck in [3] and developed later by Said in [7] with multi-spectral text images decomposition.

\section{Our Proposition: A Multiscale Handwriting Approach}

In this paper, we propose a multiscale approach to characterize and classify handwritten text based on four complementary dimensions:

- The orientation: is evaluated with a local frequencies analysis based on a hierarchical decomposition of the image area. Within this approach, pages can be seen in different scales and resolutions and allow to access different levels of information. Gabor filters have been widely used by Jain in [2] to detect specific oriented information in a document. We use, in this work, a very similar approach, based on Hermite transform. Hermite filters decompose a localized signal by a Gaussian window into a set of Hermite orthogonal polynomials [4].

Hermite filters are separable both in spatial and polar coordinates, so they can be implemented very efficiently. In the frequency domain, these filters are Gaussianlike band-pass filters and filters of increasing order analyze successively higher frequencies in the signal. They allow a local analysis which is very close to the Gabor one [6]. Figure 2.a presents the Hermite decomposition of the document of figure 1 at a given scale and up to degree 2 (lowest frequencies) as well as the reconstructed image $I_{R}$, using this decomposition, and the difference $I_{D}$ with the original image. We use image $I_{D}$ (highest frequencies) to localize the text on the page and a combination of information extracted from the four quadrants to characterize the text.

- The linearity (defined from the evolution of connected components in text lines, see figure 3). The initial image is filtered through directional bank filters (in the di- 

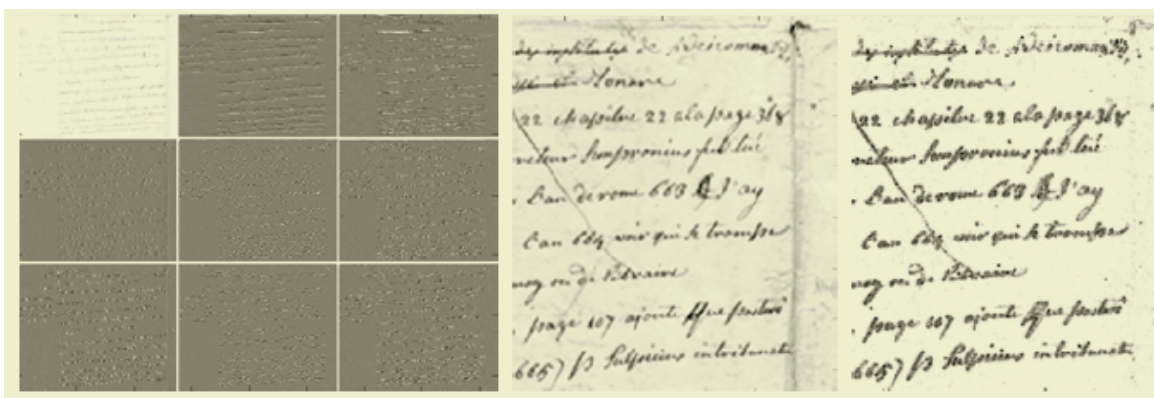

Fig. 2. Example of Hermite decomposition. (a) Hermite decomposition of the document of figure 1 at a given scale and up to degree 2 (b) a part of the reconstructed image, using decomposition of (a), (c) difference with the original image.
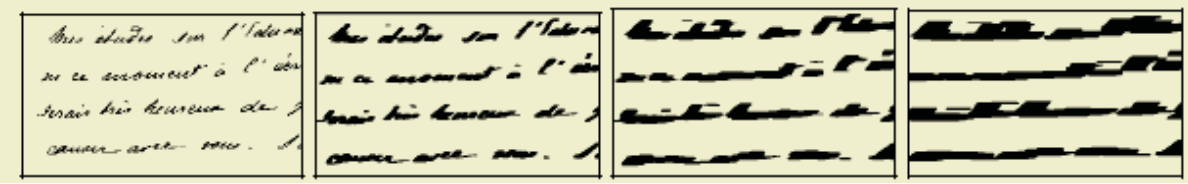

Fig. 3. Evolution of text representation with decreasing resolution and increasing line fusion.

rection of the main orientation detected with an autocorrelation analysis, see [1]) and is processed with a Difference of Offset Gaussian method (DooG). This approach is well adapted to drawings segmentation and does not need a priori knowledge of page content. The processed image reveals high frequencies in the contours of connected line drawings. We compute then an average number of connected components in the filtered image in the main direction of the drawing. The linearity reveals the size of existing connections that can be found between letters and words in a same text line. This dimension underlines visual salient differences between inter-word spaced and continuous handwritten drawings. The evolution of the linearity value through the decreasing resolutions expresses the connection rate between handwritten components of the analyzed block (from graphemes to words).

- The cursivity is evaluated as a words profile deformation. This dimension expressed the ratio that exists between the high and law words profiles (line contours), as illustrated in figure 4. The DooG approach is recursively employed to extract forms contours that are not necessary limited to words: in the first high resolutions of drawing block, the connected contours can enclose simple graphemes whereas in the low resolutions (after the process with a bank of recursive convolutions), connected contours can describe large part of a text line including one or several words. The resulting multiresolution description of contours (with high and a low profile) allows evaluating the mean curvature ratio of each considered resolution. 
- The complexity is expressed as a entropy of curve distributions and expressed the drawing density. The entropy value is proportional to the graphemes size. Its estimation is based on the number of intersections between handwritten drawing lines and oriented lines. We estimate a probability of intersections between the text and the lines and we compute for each text block the complexity measure $\mathrm{E}(\Gamma)=$ $\mathrm{pLog}(1 / \mathrm{p})=(1-\mathrm{p}) \log (1 /(1-\mathrm{p})$, where $p$ is the maximal probability of intersection . The more the text is small written, the higher is the complexity. The complexity is estimated on a handwritten text blocks perceived in different resolution.

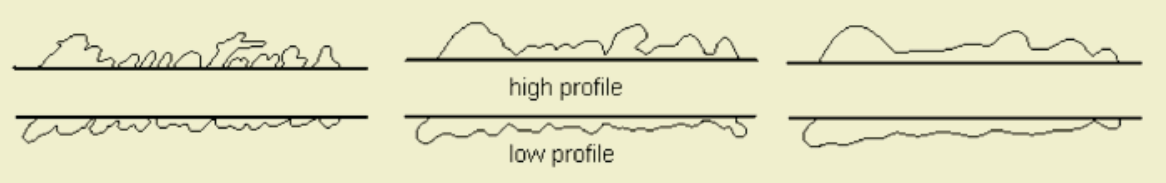

Fig. 4. High and low profiles evolution for three resolutions.

Each measure (complexity, cursivity and linearity) is then studied according to an evolution multiscale graph that simulates the perceptive behavior of a human that observes a drawing from close to far. Each feature is iteratively recomputed in successive decreasing resolution images and reported in a 2D-graph. For each graph (each dimension corresponding to a particular drawing) we estimate a single factor that expresses a unique signature of handwritten drawing lines. For the complexity, we compute the couple $\left(\mathrm{E}_{\mathrm{Min}}, \mathrm{E}_{\mathrm{Max}}\right)$ corresponding to the minimal and maximal entropy value obtained through resolution changing; for the linearity, we estimate two derivatives obtained in the decreasing curve of measures; for the cursivity, we compute a multiresolution deformation from the evolution through resolution of the high profile lengths. The resulting 2D-graphs obtained for each feature lead to the formation of visual clusters that are considered as handwriting families. Figure 5 illustrates the complexity evolution in a set of relevant handwritten text extracts.

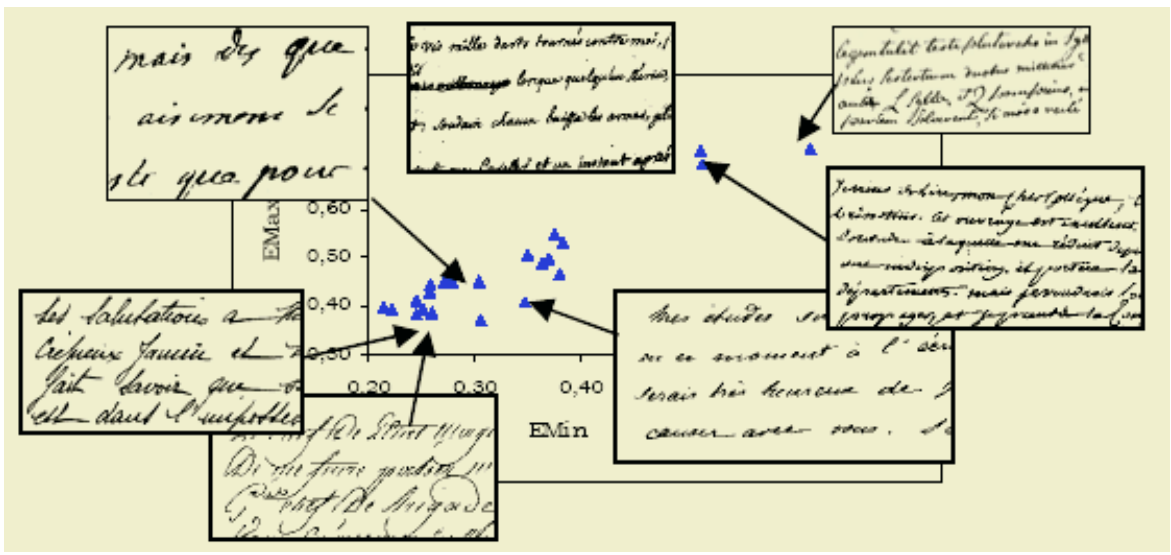

Fig. 5. Multiscale description of complexity handwriting. 
The final handwritten text block categorization leads on the combination of all individual dimensions analysed through a k-means based classification. The orientation is processed separately. Currently, we have chosen to consider 5 separable writers families for each feature. Within this approach, we are able to create 38 different families among the 50 initially considered having sometimes strong visual similarities. This approach is a robust way to show complex, compact, spaced, large or in opposite thin drawings into visual significant clusters.

\section{Conclusion and Prospective Works}

This work is a part of a project of writers' identification and is applied to Montesquieu's handwritten corpus. We have chosen to analyze the pages in their globality (with a macroscopic multi-dimensional approach of handwriting characterization, with four complementary features of orientation, complexity, curvature and linearity), and to give additional precisions on handwritten drawings with a multiscale approach. This work is currently completed with a fine writer analysis that characterizes precisely the inner writer's stability and the relevant differences between writers.

\section{References}

1. EGLIN, V., Contribution à la structuration fonctionnelle des documents, $\mathrm{PhD}$ thesis. : INSA de Lyon, 1998, 250p.

2. JAIN, A.K., BHATTACHARJEE, S., Text segmentation using Gabor filters for automatic document processing, Machine Vision \& Appli., 1992, vol.5, no.3, pp.169-184.

3. KUCKUCK, W., Writer recognition by spectra analysis, Proc. Int. Conf. In Security through Science Engineering, 1980, pp.1-3.

4. MARTENS J.-B., The Hermite transform - Theory, IEEE Trans. Acoust., Speech, Signal Processing, vol. 38, no. 9, pp. 1595-1606, 1990.

5. NOSARY, A., PAQUET, T., HEUTTE, L., Reconnaissance de textes manuscrits par adaptation au scripteur, CIFED'2002, pp.365-374.

6. RIVERO-MORENO C.J., BRES S., Conditions of similarity between Hermite and Gabor filters as models of the human visual system, In Petkov, N. and Westenberg, M.A. (eds.): Computer Analysis of Images and Patterns, Lectures Notes in Computer Science, vol. 2756, Springer-Verlag, Berlin Heidelberg, pp. 762-769, 2003.

7. SAID H. E., PEAKE G. S., TAN T. N, BAKER K. D., Writer Identification from Nonuniformly Skewed Handwriting Images, British Mach. Vision Conf., 1998. 